\title{
Anxiety and Depression During COVID-19: Are Adults in Households with Children Faring Worse?
}

\author{
Abbreviations \\ CI \\ GAD \\ OR \\ PHQ \\ $\mathrm{PP}$ \\ U.S.
}

\author{
Confidence interval \\ Generalized Anxiety Disorder \\ Odds ratio \\ Patient Health Questionnaire \\ Predicted probability \\ United States
}

Analyses. We performed logistic regression (Stata V15.1, College Station, TX). Covariates included age, race, Hispanic ethnicity, education, income, marital status, and household size. We included an interaction term for household-with-children status by sex and calculated predicted probabilities (PP).

J Gen Intern Med 37(5):1328-30

DOI: $10.1007 / \mathrm{s} 11606-021-07256-9$

(c) Society of General Internal Medicine 2021

\section{INTRODUCTION}

U.S. adults have experienced a threefold increase in anxiety and depression symptomatology during COVID-19. ${ }^{1}$ Caregivers of children reported elevated parenting stress ${ }^{2,3}$, food insecurity ${ }^{3}$, and economic worries. ${ }^{4}$ Many struggled to manage childcare and home education. ${ }^{3,5}$ However, prior studies ${ }^{2-}$ ${ }^{5}$ used non-representative samples and lacked comparison to non-caregivers. In this brief report, we hypothesized that (H1) adults in households with children would be more likely to screen positive for anxiety and depression. Because women report more mental health symptoms than men ${ }^{1,6},(\mathrm{H} 2)$ females in households with children would be especially likely to screen positive for anxiety and depression.

\section{METHODS}

The U.S. Census Household Pulse Survey, Phase I, collected data weekly from April 23, 2020, to July 21, 2020. Exclusion criteria were as follows: (1) $\geq 65$ years; (2) missing anxiety/ depression data $(N=592,545)$.

Anxiety and Major Depression. Anxiety and major depression were measured with Generalized Anxiety Disorder-2(GAD-2) and Patient Health Questionnaire-2 (PHQ-2). Summed scores $\geq 3$ were the cutoff for potential anxiety and depressive disorders.

Households with Children. Adults aged 18-64 living with at least one child $<18$ years.

Received July 12, 2021

Accepted October 28, 2021

Published online January 4, 2022

\section{RESULTS}

The average age of respondents was 44.65 (range: 18-64;SD $=11.72$ ); $58 \%$ married; $62 \%$ female; $80 \%$ White, $9 \%$ Black, 5\% Asian, 5\% other race; $10 \%$ Hispanic ethnicity. Average household size was 3 (range: $1-10 ; S D=1.59$ ). The average percentage of positive screens for anxiety and depression were $35 \%$ and $24 \%$ across the 12 weeks.

Positive screens for anxiety and depression increased over time in a nonlinear fashion and were best fit using quadratic models (Figs. 1 and 2). While females were more likely than males to screen positive for anxiety at all time points (Fig. 1), household-with-children status had no effect on anxiety (OR $=0.99,95 \%$ CI $[0.97,1.00])$. Respondents who were male $(\mathrm{OR}=0.70,95 \%$ CI $[0.69,0.71])$, married $(\mathrm{OR}=0.85,95 \%$ CI $[0.84,0.86])$, and those with higher income (OR $=0.87$, $95 \%$ CI $[0.87,0.88])$ were less likely to screen positive for anxiety. Larger household size was a weak risk factor (OR = $1.01,95 \%$ CI $[1.01,1.02])$. Household-with-children status was associated with lower probability of screening positive for anxiety for females (no children PP: 0.38, children PP: 0.37, $\chi^{2}(1)=24.78, p<0.001$ ); but males in households with children had slightly greater probability of screening positive (no children PP: 0.30 , children PP: $0.31, \chi^{2}(1)=12.73, p<$ 0.001) (Fig. 1).

Household-with-children status was associated with decreased positive screens for depression (OR $=0.85,95 \% \mathrm{CI}$ $[0.83,0.86])$, as was being male $(\mathrm{OR}=0.89,95 \%$ CI $[0.87$, $0.90])$, being married $(\mathrm{OR}=0.76,95 \% \mathrm{CI}[0.75,0.77])$, and higher income (OR 0.84, 95\% CI [0.83, 0.84]). Larger household size was a weak risk factor $(\mathrm{OR}=1.02,95 \%$ CI $[1.01$, 1.02]. Household-with-children status was associated with lower probability of screening positive for depression, for both females (no children PP: 0.27 , children PP: $0.23, \chi^{2}(1)=$ 449.81, $p<0.001$ ) and males (no children PP: 0.24, children PP: $\left.0.22, \chi^{2}(1)=59.35, p<0.001\right)$ (Fig. 2). 


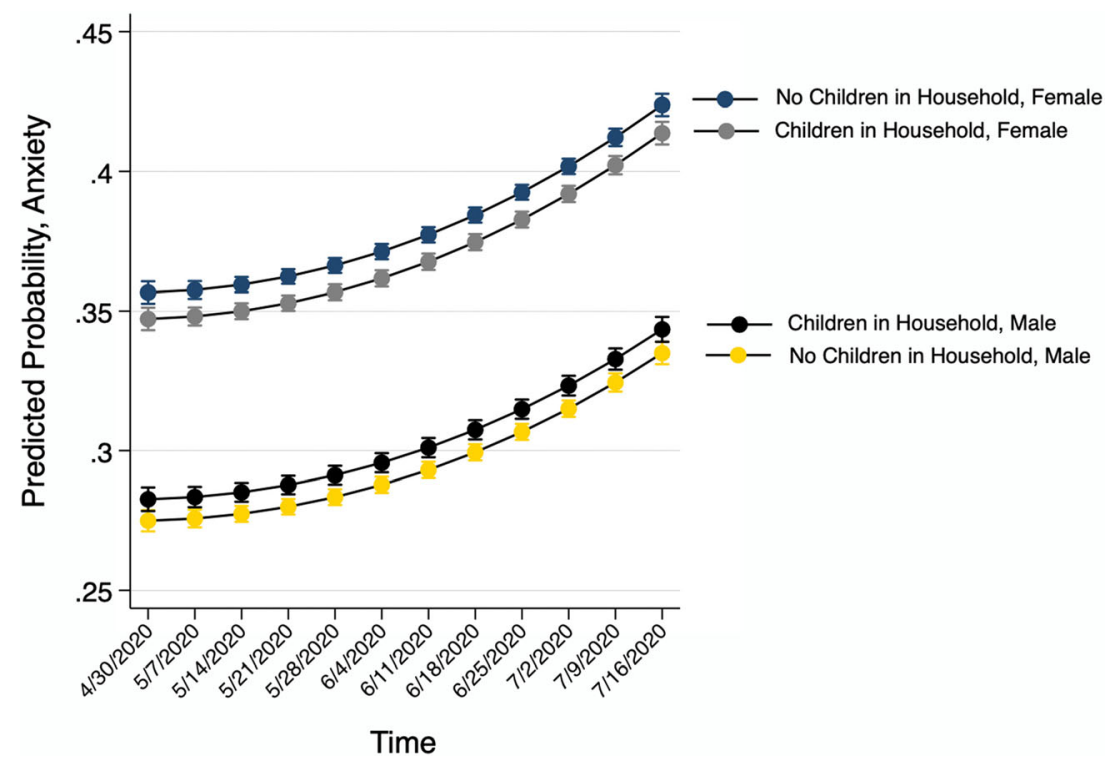

Figure 1 Graph of predicted probabilities for anxiety by household-with-children status and sex. Phase I of the U.S. Census Household Pulse Survey included surveys of U.S. households conducted weekly beginning with Wave 1 (April 2020) and concluding with Wave 12 (July 2020 ). Analytic sample $N=592,545$. Models controlled for age, race, Hispanic ethnicity, education, income, marital status, and household size.

\section{DISCUSSION}

Overall, caregiving and gender appear to have differential associations with pandemic mental health. Contrary to hypotheses, adults in households with children did not experience heightened depression symptomatology. Child caretaking-beyond the effect of larger household size - may reduce depression symptomatology during pandemic social isolation.

However, compared to males not living with children, males in households with children experienced slightly heightened anxiety symptomatology. Men have experienced greater increase in mental health symptoms during COVID-19 than women ${ }^{6}$. Male caregivers may be vulnerable to pandemic worries, e.g., employment loss. In addition to screening adults, internal medicine physicians may need to attend to heightened anxiety among males living with children during the pandemic.

We note study limitations. GAD-2 and PHQ-2 screening tools are not substitutes for diagnostic measures. We cannot ascertain whether all respondents in households with children were active caregivers. The Pulse surveys asked about symptomatology over 1 week rather than 2 weeks; thus, estimates herein may be conservative. ${ }^{6}$

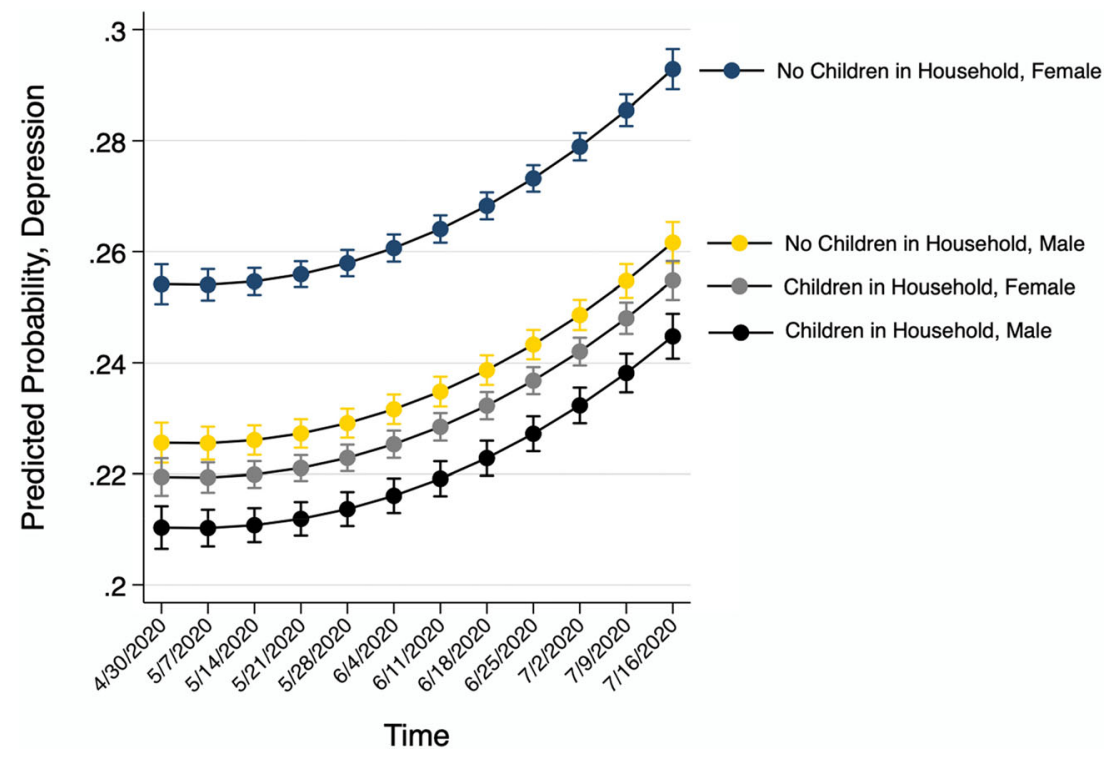

Figure 2 Graph of predicted probabilities for depression by household-with-children status and sex. Phase I of the U.S. Census Household Pulse Survey included surveys of U.S. households conducted weekly beginning with Wave 1 (April 2020) and concluding with Wave 12 (July 2020). Analytic sample $N=\mathbf{5 9 2 , 5 4 5}$. Models controlled for age, race, Hispanic ethnicity, education, income, marital status, and household size. 
Shawna J. Lee, $P h D^{1}$

Kaitlin P. Ward, $M S W^{1}$

Andrew Grogan-Kaylor, $\mathrm{Ph} \mathrm{D}^{1}$

Vijay Singh, $M D^{2}$

${ }^{1}$ School of Social Work, University of Michigan, Ann Arbor, MI, USA

${ }^{2}$ Departments of Internal Medicine, Emergency Medicine, and Family Medicine, Medical School, University of Michigan,

Ann Arbor, MI, USA

Corresponding Author: Shawna J. Lee, PhD; School of Social Work, University of Michigan, Ann Arbor, MI, USA (e-mail: shawnal@umich.edu).

\section{Declarations:}

Conflict of Interest: The authors declare that they do not have a conflict of interest.

Institutional Review Board: This study consisted of secondary anal ysis of deidentified Census data and was exempt from oversight by the University of Michigan Institutional Review Board (HUM00191066).

\section{REFERENCES}

1. Twenge JM, Joiner TE. U.S. Census Bureau-assessed prevalence of anxiety and depressive symptoms in 2019 and during the 2020 COVID-19 pandemic. Depression \& Anxiety. 2021;37:954-956.

2. Freisthler B, Gruenewald PJ, Tebben E, McCarthy KS, Wolf JP. Understanding at-the-moment stress for parenting during COVID19stay-at-home restrictions. Social Science \& Medicine. 2021 (279):114025.

3. Patrick SW, Henkhaus LE, Zickafoose JS, et al.Well-being of parents and children during the COVID-19 pandemic: A national survey. Pediatrics. 2020; 146(4):e2020016824.

4. Rodriguez CM, Lee SJ, Ward KP, Pu DF. The perfect storm: Hidden risk of child maltreatment during the COVID-19 pandemic. Child Maltreatment. 2021;26(2):139-151.

5. Lee SJ, Ward KP, Chang OD, Downing K. Parenting activities and the transition to home-based education during the COVID-19 pandemic. Child Youth Serv Rev. 2021;122:105585.

6. Cai C, Woolhandler S, Himmelstein DU, Gaffney A. Trends in Anxiety and Depression Symptoms During the COVID-19 Pandemic: Results from the US Census Bureau's Household Pulse Survey. Journal of General Internal Medicine. 2021;36(6): 1841-1843.

Publisher's Note Springer Nature remains neutral with regard to jurisdictional claims in published maps and institutional affiliations. 